\title{
Keine Chance den Trojanern
}

\author{
Die Maschen von Cyberkriminellen werden immer ausgeklügelter. \\ Im Trend liegen Erpressungstrojaner, welche die kompletten Da- \\ ten auf den infizierten Rechnern verschlüsseln. Von den Attacken \\ sind längst auch Ärzte betroffen. Praxen können vorbeugen.
}

Ob „Locky“ oder „TeslaCrypt“: Derzeit machen Verschlüsselungs-Trojaner die Runde. Was das für den Praxisbetrieb bedeuten kann, musste kürzlich ein Internist erfahren: „Stellen Sie sich vor, Sie arbeiten in Ihrer großen Praxis mit mehreren Kollegen und vielen Mitarbeitern, es ist viel los (...) und plötzlich stürzen im Minutentakt die einzelnen Rechner von 18 Arbeitsplätzen ab. Man hofft auf den herbeieilenden Netzwerkadministrator, und der sagt schlicht: ,Herr Doktor, Sie haben ein größeres Problem. Soeben wurden Ihre Praxisdaten verschlüsselt.“ Damit liegt der Praxisbetrieb zumindest teilweise lahm. Schuld ist hier die sog. „Ransomware“. Damit sind Schadprogramme gemeint, die den Zugang zum Computer oder zu mobilen Geräten verhindern oder aber wie im Fall dieses Internisten die gespeicherten Daten verschlüsseln. Übertragen werden die Schadprogramme über E-Mail-Anhänge und mitgeschickte Links (in beiden Fällen meist als eilige Rechnung getarnt) oder aber über angebliche Software-Updates.

Wie ausgeklügelt und vielfältig die Tricks der Cyberkriminellen mittlerweile sind, zeigt der Bericht des Bundesamtes für Sicherheit in der Informationstechnologie (BSI) zur IT-Sicherheit in Deutschland für 2015. Auf über 439 Millionen schätzt das BSI die Gesamtzahl der Schadprogrammvarianten. Dabei würden sich immer mehr dieser Varianten automatisch während der Weiterverbreitung auf den Rechnern generieren. Aufgrund seines hohen Marktanteils sei zwar hauptsächlich das Betriebssystem Windows betroffen, da Eintrittstore aber oft der Adobe Flash Player und eben die bereits erwähnten E-Mail-Anhänge sind, können auch Besitzer von Apple-Rechnern betroffen sein.

Einbußen im fünfstelligen Bereich Die Erpresser präsentieren sich „dreist und frech“, wie der internistische Kollege weiter erzählte, mit einem auf der Festplatte abgelegten Schreiben. Gegen Be- zahlung in Bitcoins - einer digitalen Währung - würde die Freischaltung der verschlüsselten Daten veranlasst. Doch weil es keine Garantie dafür gibt, dass die Daten anschließend auch tatsächlich entschlüsselt werden, hatte sich zumindest der Internist dazu entschlossen, nicht zu zahlen. Der Plan: Das Team wollte die Praxisdaten selbst rekonstruieren. „Was selbstverständlich mit einem erheblichen finanziellen und zeitlichen Aufwand verbunden ist. Hinzu kommt der Umsatzausfall für circa drei geschlossene Praxistage - ohne EDV können moderne Praxen nicht mehr betrieben werden. Hier sind schnell fünftstellige Einbußen erreicht", berichtete der Internist.

\section{Datensicherung und zwar mit Mehrfachschutz}

Das Stichwort lautet Datensicherung. Dazu rät auch das BSI eindringlich, gerade wegen der aktuell gehäuften Fälle von Ransomware-Attacken auf kleinere Betriebe und Privatpersonen. Im letzten Jahr hat es aber auch das System eines Klinikkonsortiums getroffen. Hier hatte „Cryptowall“ zugeschlagen. Weil in großen Kliniken die Datensicherung meist ein Standardprozess ist, konnte der Datenausfall jedoch auf zwölf Stunden begrenzt werden. Aber: Ein weiterer finanzieller Schaden sei nicht ausgeschlossen, heißt es, da Abrechnungen unter Umständen nicht mehr nachvollzogen werden könnten.

Dabei sind die Spielregeln für die Datensicherung einfach:

- Sie sollte regelmäßig (je nach Praxisgröße täglich bis wöchentlich) auf einem externen Speichermedium vorgenommen werden. Hierzu eignen sich etwa gute RAID(„redundant array of independent disks)-Systeme. Dahinter verbirgt sich ein Verbund unabhängiger Festplatten in einem Gehäuse. Die Daten werden beim Speichern gleichzeitig auf mehreren Festplatten abgelegt. Das senkt das Risiko, dass sie in irgendeiner Form nicht auslesbar sind.

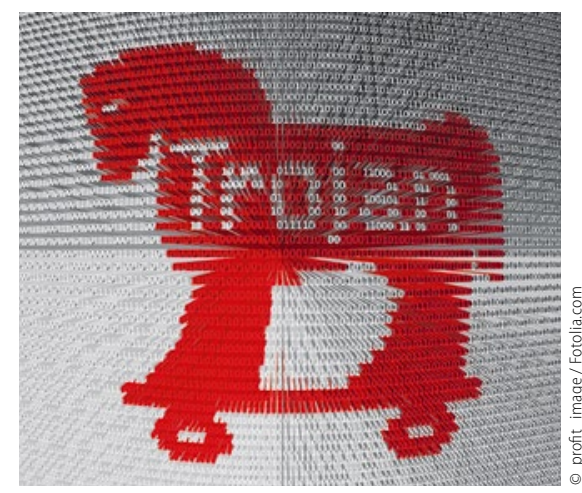

In kleinen Praxen kann aber auch eine normale USB-Festplatte für die Datensicherung genutzt werden.

- Die Datensicherung sollte immer getrennt vom Rechnersystem der Praxis aufbewahrt und vor allem nicht dauerhaft an dieses angeschlossen werden, denn: „Viele Verschlüsselungstrojaner können auch Daten auf externen und Netzwerklaufwerken unbrauchbar machen", mahnt das BSI.

- Außerdem sollte das Praxisteam anhand einiger ausgewählter Dateien prüfen, ob sich die gesicherten Dateien tatsächlich wiederherstellen lassen und die Datensicherung funktioniert.

Am besten das ganze Programm Zusätzlich benötigen Praxen diverse Schutzmechanismen, die Eindringlinge von vornherein abwehren. Dazu zählen in jedem Fall eine aktivierte, aktuelle Firewall (die meisten Internetrouter haben bereits eine integrierte Firewall), ein aktuell gehaltener Antivirenscanner sowie ein Betriebssystem und einen Internetbrowser, die durch regelmäßige Updates dem neuesten Sicherheitsstandard entsprechen. Wichtig ist laut BSI darüber hinaus eine gesunde Portion Misstrauen gegenüber unbekannten E-MailAbsendern, etwa mit Rechnungsanhang.

Gleiche Vorsicht gelte bei E-Mail-Faxen. Beim Surfen im Internet hilft es, wenn der Virenscanner sichere und unsichere Websites symbolisch kennzeichnet. Praxen müssen außerdem die Schweigepflicht auch für die Patientendaten auf den Rechnern beachten. Es gilt daher, die Systeme - falls der Nachweis doch einmal strafrechtlich nötig ist nach bestem Wissen durch den aktuellen Stand der Technik zu schützen.

Der eingangs erwähnte Internist geht mittlerweile sogar noch einen Schritt weiter: „Der Mailrechner wird vom Netz und vom Server abgehängt; nur dort lokal geprüfte Mails werden weiter geleitet.“

RebekkaHöhl 